\section{Autistic peer-to-peer information transfer is highly effective}

\author{
Catherine J Crompton' (D), Danielle Ropar'2, \\ Claire VM Evans-Williams ${ }^{3}$, Emma G Flynn ${ }^{4}$ \\ and Sue Fletcher-Watson' ${ }^{1}$
}

Autism

2020, Vol. 24(7) 1704-1712

(C) The Author(s) 2020

(c) (i) \$

Article reuse guidelines:

sagepub.com/journals-permissions DOI: $10.1177 / 1362361320919286$

journals.sagepub.com/home/aut

(S)AGE

\begin{abstract}
Effective information transfer requires social communication skills. As autism is clinically defined by social communication deficits, it may be expected that information transfer between autistic people would be particularly deficient. However, the Double Empathy theory would suggest that communication difficulties arise from a mismatch in neurotype; and thus information transfer between autistic people may be more successful than information transfer between an autistic and a non-autistic person. We investigate this by examining information transfer between autistic adults, non-autistic adults and mixed autisticwith-non-autistic pairs. Initial participants were told a story which they recounted to a second participant, who recounted the story to a third participant and so on, along a 'diffusion chain' of eight participants $(n=72)$. We found a significantly steeper decline in detail retention in the mixed chains, while autistic chains did not significantly differ from non-autistic chains. Participant rapport ratings revealed significantly lower scores for mixed chains. These results challenge the diagnostic criterion that autistic people lack the skills to interact successfully. Rather, autistic people effectively share information with each other. Information transfer selectively degrades more quickly in mixed pairs, in parallel with a reduction in rapport.
\end{abstract}

\title{
Lay abstract
}

Sharing information with other people relies on the ability to communicate well. Autism is defined clinically by deficits in social communication. It may therefore be expected that autistic people find it difficult to share information with other people. We wanted to find out whether this was the case, and whether it was different when autistic people were sharing information with other autistic people or with non-autistic people. We recruited nine groups, each with eight people. In three of the groups, everyone was autistic; in three of the groups, everyone was non-autistic; and three of the groups were mixed groups where half the group was autistic and half the group was non-autistic. We told one person in each group a story and asked them to share it with another person, and for that person to share it again and so on, until everyone in the group had heard the story. We then looked at how many details of the story had been shared at each stage. We found that autistic people share information with other autistic people as well as non-autistic people do with other non-autistic people. However, when there are mixed groups of autistic and non-autistic people, much less information is shared. Participants were also asked how they felt they had got on with the other person in the interaction. The people in the mixed groups also experienced lower rapport with the person they were sharing the story with. This finding is important as it shows that autistic people have the skills to share information well with one another and experience good rapport, and that there are selective problems when autistic and non-autistic people are interacting.

\section{Keywords}

adults, communication and language, psychological theories of autism, social cognition and social behaviour

\section{Introduction}

Autism spectrum disorder (hereafter 'autism', Kenny et al., 2016) is clinically characterised by lifelong impairments in social and communication domains, the presence of restricted and repetitive behaviours and sensory perceptual features (American Psychiatric Association (APA), 2013). A large body of research indicates that autistic people struggle with attributing mental states to others and

\footnotetext{
'The University of Edinburgh, UK

${ }^{2}$ The University of Nottingham, UK

${ }^{3}$ The Autism Academy, UK

${ }^{4}$ Queen's University Belfast, UK

Corresponding author:

Catherine J Crompton, Division of Psychiatry, Centre for Clinical Brain Sciences, The University of Edinburgh, Kennedy Tower, Royal Edinburgh Hospital, Morningside Terrace, Edinburgh EHIO 5HF, UK. Email: catherine.crompton@ed.ac.uk
} 
identifying social cues such as basic and complex facial emotions (Baron-Cohen et al., 1997), tone of voice (Rutherford \& Baron-Cohen, 2002), sarcasm (Persicke et al., 2013) and social faux pas (Baron-Cohen et al., 1999) compared with non-autistic people, resulting in difficulties in social interactions.

However, non-autistic individuals have communicative difficulties when interacting with autistic individuals. Non-autistic people struggle to identify autistic mental states (Edey et al., 2016), identify autistic facial expressions (Sheppard et al., 2016), overestimate autistic egocentricity (Heasman \& Gillespie, 2018), and are less willing to socially interact with autistic people (Sasson et al., 2017). Thus, although non-autistic people are generally characterised as socially skilled, these skills may not be functional, or effectively applied, when interacting with autistic people. This bi-directional disconnect in communication and understanding between autistic and non-autistic people has been labelled the 'double empathy problem' (Milton, 2012; Milton et al., 2018). One implication of the 'double empathy problem' is that if autistic 'social impairments' result from a mismatch between autistic and nonautistic populations, they may disappear in within-group interactions. Thus, predictions based on clinical definitions of autism about autistic-autistic interaction would be that they would be non-functional or ineffective, and in contrast, predictions based on the Double Empathy theory about autistic-autistic interaction would be that they would be successful and positive. However, to date, there is no experimental evidence directly testing whether autisticautistic interactions are successful.

Recent research has found that autistic people prefer interacting with other autistic people, and experience close social affiliation with them (Crompton et al., 2020; Morrison et al., 2019), and has begun to examine the mechanisms that underlie this preference. Autistic people are, by definition, more familiar with autism, and are more accurate at correctly detecting the intentions of autistic people than non-autistic people (Heasman \& Gillespie, 2018; Sheppard et al., 2016). Autistic people report exhibiting fewer autistic traits when interacting with other autistic people compared with non-autistic people (Gernsbacher et al., 2017) and are less deterred by negative first impressions of autistic adults in deciding whether to later socialise with them (DeBrabander et al., 2019). In addition, autistic interactions follow a distinctive pattern of intersubjectivity, which while unconventional by non-autistic standards, function effectively to facilitate mutual understanding (Heasman \& Gillespie, 2019a). Collectively, this evidence suggests that autistic interactions may be unique, but similarly facilitative of communication as non-autistic interactions. However, to date, not research has looked at information transfer as a measure of communicative success, contrasting how autistic and non-autistic people share information with their autistic and non-autistic peers.
This study directly compares how autistic and nonautistic people interact when in matched (same diagnostic status) or mixed (autistic with non-autistic pairs), in an information-sharing context. Aligned with the double empathy theory, we hypothesised that information transfer would yield higher fidelity for sequences of matched pairs (whether all autistic or all non-autistic), and that there would be poorer quality information transfer for mixed pairs of autistic and non-autistic people. We also hypothesised that, alongside information transmission, self-rated rapport would be higher for matched pairs (whether all autistic or all non-autistic), and that mixed pairs would experience lower interactional rapport.

Information transfer was operationalised using a 'diffusion chain' technique (Bartlett, 1932) (Figure 1) which involves examining the fidelity of information as it is passed along linear 'chains' of participants. By assessing and analysing the rate which information degrades along the chain, the efficacy of information transfer can be explored (Mesoudi \& Whiten, 2008). This type of diffusion chain paradigm is novel to the field of autism research.

\section{Methods}

This study was carried out in accordance with the British Psychological Society's Code on Human Research Ethics. Experimental procedures were reviewed and approved by the University of Edinburgh Psychology Research Ethics Committee, and all participants provided written informed consent prior to participating in the study. Participants were remunerated for their time.

\section{Study design}

The article describes a between-groups experimental study, comparing information-sharing outcomes using a diffusion chain paradigm, comparing autistic, non-autistic and mixed groups.

\section{Participants}

A total of 72 adult volunteers participated, with 24 adults in each of the autistic, non-autistic and mixed sets. $\mathrm{G}^{*}$ power was used to run a prospective power analysis, indicating $95 \%$ power to detect a medium effect of 0.5 at the standard 0.05 alpha error probability with a sample size of 66. To allow us to have an even number of eightperson diffusion chains in each group, 72 participants were recruited through community networks, social media and local autism organisations.

The three groups were matched on age, gender, years of education and intelligence quotient (IQ) (Tables 1 and 2). All spoke English to a native level and did not have a clinical diagnosis of social anxiety disorder. All non-autistic participants scored less than 32 on the Autism Quotient 


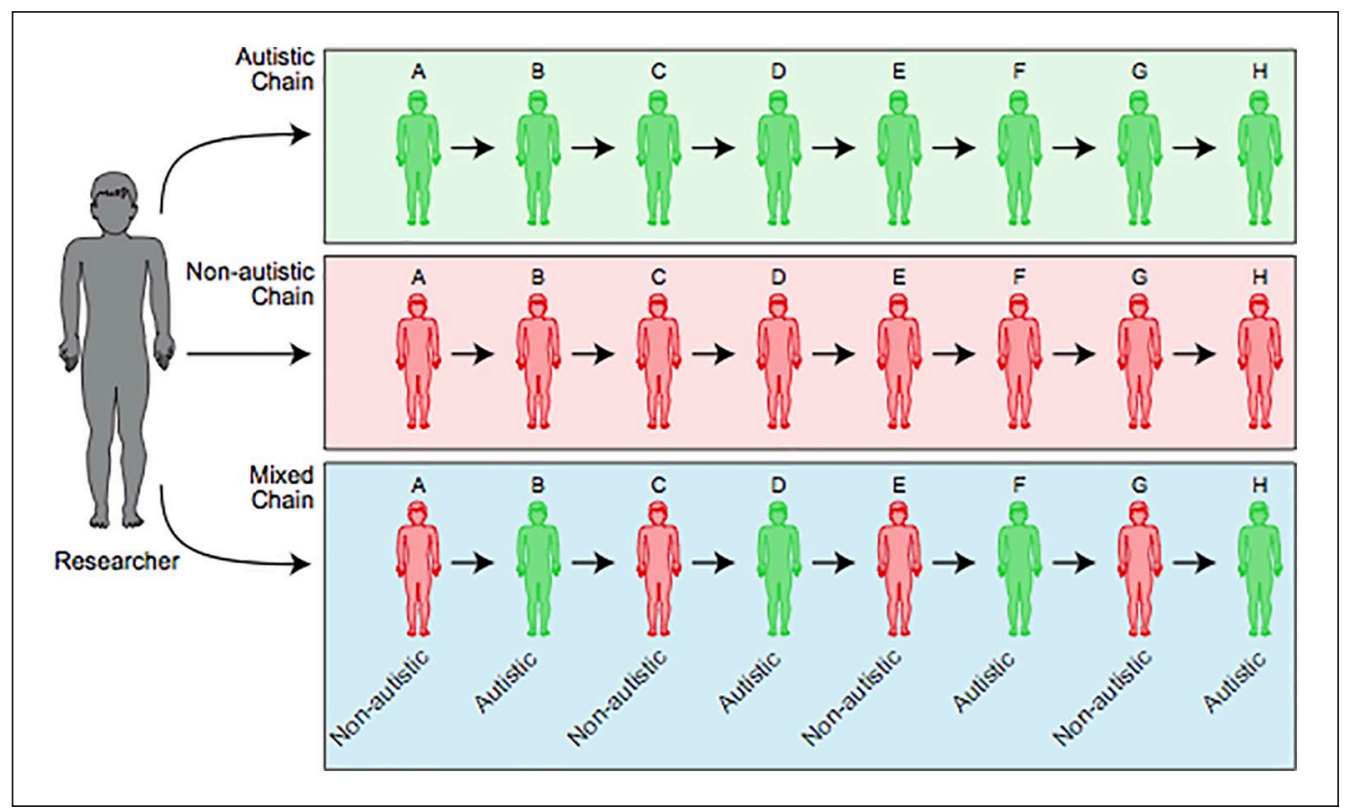

Figure I. Illustration of the diffusion chain technique.

Table I. Descriptive statistics (mean (standard deviation) on demographics by diagnostic status, using independent $t$-test and Fisher's exact test comparisons.

\begin{tabular}{|c|c|c|c|}
\hline & Non-autistic & Autistic & Comparisons \\
\hline Age & $36.31(13.00)$ & $37.36(12.59)$ & $t(69.93)=0.35, p=0.73$ \\
\hline Gender & $29 \mathrm{~F}, 7 \mathrm{M}$ & $28 \mathrm{~F}, 5 \mathrm{M}, 3 \mathrm{NB}^{\mathrm{a}}$ & Fishers exact test $p=0.24$ \\
\hline Years of education & I7.74 (I.8I) & $17.19(2.45)$ & $t(64.44)=-1.07, p=0.29$ \\
\hline IQ - WASI-II & II 5.86 (I0.7I) & II 5.64 (I7.03) & $t(58.92)=-0.67, p=0.95$ \\
\hline Autism Quotient & $\begin{array}{r}13.67(5.87) \\
\text { Range }=3-28\end{array}$ & $\begin{array}{c}36.78(6.60) \\
\text { Range }=25-47\end{array}$ & $t(69.07)=15.69, p<0.000 \mid$ \\
\hline Age of diagnosis & $\mathrm{N} / \mathrm{A}$ & $30.66(11.81)$ & $\mathrm{N} / \mathrm{A}$ \\
\hline
\end{tabular}

aNon-binary.

'Wechsler Abbreviate Scale of Intelligence - II.

Table 2. Descriptive statistics (mean (standard deviation) on demographic for assigned sets, using Kruskal-Wallis chi square, ANOVA and Fisher's exact test comparisons.

\begin{tabular}{|c|c|c|c|c|}
\hline & Non-autistic & Autistic & Mixed & Comparisons \\
\hline Age & $37.92(14.39)$ & $37.33(13.13)$ & $35.25(10.76)$ & $X^{2}(2)=0.27, p=0.87$ \\
\hline Gender & $2 \mid F, 3 M$ & $18 \mathrm{~F}, 3 \mathrm{M}, 3 \mathrm{NB}^{\mathrm{a}}$ & $18 \mathrm{~F}, 6 \mathrm{M}$ & Fisher's exact test $p=0.17$ \\
\hline Years of education & I $7.83(1.52)$ & I $7.44(2.80)$ & $17.12(1.98)$ & $X^{2}(2)=1.83, p=0.40$ \\
\hline IQ - WASI-II & II 5.04 (II.78) & I I 4.42 (I6.89) & II 7.79 (I 3.62$)$ & $F(2,69)=0.38, p=0.68$ \\
\hline Age of diagnosis & NA & $30.55(12.72)$ & $30.89(10.20)$ & $X^{2}(I)=0.36, p=0.85$ \\
\hline
\end{tabular}

ANOVA: analysis of variance.

aNon-binary.

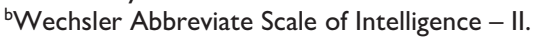

(AQ), indicating low levels of autistic traits (Baron-Cohen et al., 2001). All autistic participants were either clinically diagnosed $(n=33)$, or if self-diagnosed $(n=3)$ they scored above 72 on the Ritvo Autism-Aspergers Diagnostic Scale - Revised (RAADS-R). A RAADS-R score of above 65 is consistent with a clinical diagnosis of autism Ritvo et al., 2011. In addition to this, all self-diagnosed autistic participants scored above 32 on the AQ indicating high levels of autistic traits (Baron-Cohen et al., 2001). Participants also completed the Wechsler Abbreviated Scale of Intelligence 
II (WASI-II) (Wechsler, 2011), a measure of IQ, with all participants scoring within a typical range. Demographics for the autistic and non-autistic participants are reported in Tables 1 and 2).

\section{Procedure}

Diffusion chains. The experiment took place in a research suite at the Division of Psychiatry at the University of Edinburgh. Seventy-two participants were divided into three equal sets: non-autistic (N), autistic (A) and mixed (M). Each set was divided further into three diffusion chains of eight people: nine diffusion chains in total. Each of the nine diffusion chains was run on separate 'Research Days', which eight participants attended at a time. Participants were assigned to one of nine Research Days based on their diagnostic status (autistic or non-autistic), age, gender and years of education.

Participants were part of eight-person chains, where either all participants were autistic, all were non-autistic or four were autistic and four non-autistic. In this last case, the chain alternated between the two, starting with a nonautistic participant. Each of the chains was ordered in ascending age order, to minimise any potential effects of age-related memory decline, with minimal switches by gender (see Supplementary Table 1). Participants were aware whether they were in an autistic, a non-autistic or a mixed chain. Participants did not meet before the study started, and were isolated in separate rooms throughout the study, except when participating in the diffusion chain.

The story used in the diffusion chains was a 30-point story, which followed a bear on a surreal adventure (Crompton \& Fletcher-Watson, 2019). The story was designed to be difficult to predict, and did not include any inherently social aspects. The story had a Flesch-Kincaid Grade Level of 4.4, equivalent to age 9-10 reading level and a Flesch Reading Ease score of 90.1; a score of 60 or more is considered easy to read (Flesch, 1948; Kincaid et al., 1975).

The researcher read the story to the first participant (A). The researcher left the room, and a second participant (B) entered. A then recounted the story to B. A left the room, and a third participant $(\mathrm{C})$ entered. $\mathrm{B}$ then recounted the story to $\mathrm{C}$ and so on, to the eighth participant $(\mathrm{H})$. The eighth participant recounted the story aloud alone. Participants waited in separate rooms for their turn, to avoid contamination during the information sharing. All mixed chains started with a non-autistic participant. Diffusion chains were video recorded for scoring purposes. Participants' final score corresponded to the number of story details they had passed on to the next person in the chain.

Rapport. After completing the diffusion chain, participants indicated their feelings of rapport with their neighbours in the chain using a 100-point scale with five dimensions: ease, enjoyment, success, friendliness and awkwardness (reverse

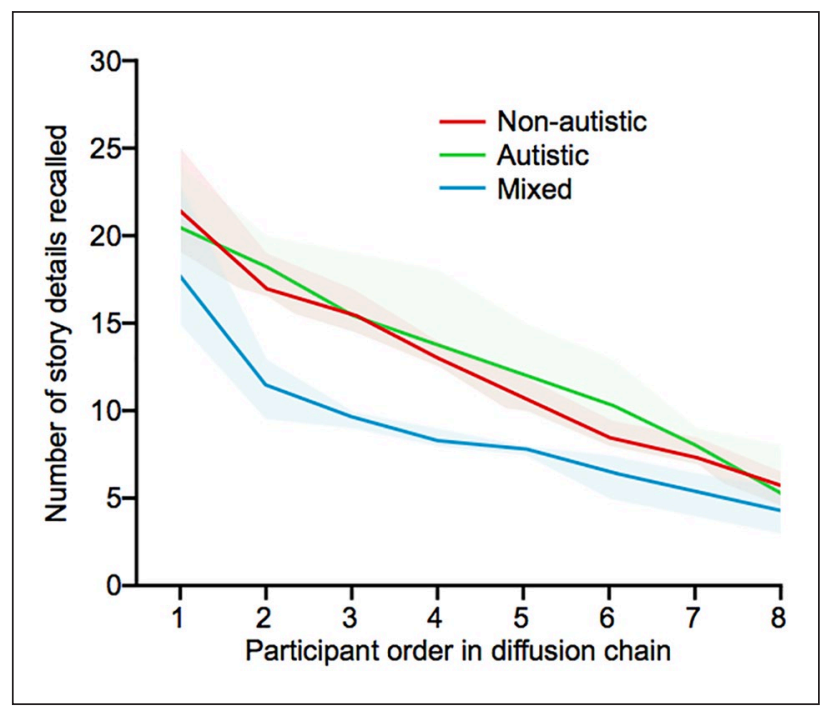

Figure 2. Mean and range of story details (out of 30) transferred in the diffusion chain, by group and position.

scored). The five dimensions had a Cronbach's alpha of 0.71 , and so were summed to create a single scale of interactional rapport. Subsequent Bayesian analyses were calculated in JASP (JASP Team, 2019).

\section{Results}

Initial pre-registered (Crompton \& Fletcher-Watson, 2019) analyses tested for chain-type differences in the overall number of story details (out of a total of 30, averaged across all participants in each chain type regardless of position) recalled using analysis of variance (ANOVA), revealing significant group differences $(F(2,69)=4.60$, $p<0.05$ ). Post hoc contrasts indicated that the non-autistic and autistic chains did not differ from each other $(\mathrm{MeanN}=12.40, \mathrm{SDN}=5.28 ; \mathrm{MeanA}=12.96, \mathrm{SDA}=5.31$, Tukey honestly significant difference (HSD) $p=0.92$ ), though both groups recalled significantly more details than the mixed chains (MeanA-N=8.92, SDA-N $=4.34$; Tukey HSDN and A-N $p=0.04$, TukeyA and A-N HSD $p=0.01$ ).

Regression analysis was carried out to investigate whether number of details recalled deteriorated at a comparable rate for the three chain types (Figure 2, see Supplementary Figure 1 for individual chains). Predictors were chain type (autistic, non-autistic and mixed, with non-autistic as the reference group), and position in the chain (1-8), and included an interaction of chain type and position in chain. Individual data were entered without first being averaged across three chains of the same type. There was a steeper decline for the mixed chains $(b=-6.04$, standard error $(S E)=1.32, p<0.0001)$, while autistic chains' recall did not differ to that of chains of non-autistic people ( $b=0.13, S E=1.32, p=0.93$ ), see Table 3. Position also significantly predicted the amount of information 
Table 3. Regression of the effect of chain type and order in chain on overall accuracy of data transfer between participants.

\begin{tabular}{|c|c|c|c|c|}
\hline & Estimate & Standard error & $t$ & $p$ \\
\hline Intercept (non-autistic chains) & 22.10 & 0.94 & 23.61 & $<0.000 I^{*}$ \\
\hline \multicolumn{5}{|l|}{ Main effects } \\
\hline Group: autistic chains & 0.13 & 1.32 & 0.09 & 0.9251 \\
\hline Group: mixed chains & -6.04 & 1.32 & -4.56 & $<0.000 I^{*}$ \\
\hline Order in chain & -2.15 & 0.18 & -11.63 & $<0.000 I^{*}$ \\
\hline \multicolumn{5}{|l|}{ Interactions } \\
\hline Group: autistic $\times$ order in chain & 0.09 & 0.26 & 0.37 & 0.71 \\
\hline Group: mixed $\times$ order in chain & 0.57 & 0.26 & 2.17 & $0.03 *$ \\
\hline
\end{tabular}

DF: degrees of freedom.

Residual standard error: 2.08 on 66 degrees of freedom. Multiple R-squared =0.85; adjusted R-squared $=0.84$. F statistic: 77.05 on 5 and 66 DF. $*_{p}<0.0001$.

recalled $(b=-2.15, S E=0.18, p<0.0001)$, and together chain type and position accounted for $85 \%$ of the variance in amount of information recalled $(F(5,66)=77.05$, $p<0.0001, R 2=0.85)$. In addition, a significant interaction between being in an mixed chain and the order of participation was found, indicating that the rate that detail recall deteriorated in the mixed chains was significantly faster $(b=0.57, S E=0.26, p<0.05)$. Thus, autistic and non-autistic chain types do not differ in their informationsharing abilities, and selective penalties for information transfer occur when there is a diagnostic mismatch.

As shown in Figure 2, the mean number of details recalled by the first person in a mixed chain was lower than for other chains. Supplementary Table 2 describes the first participant in each diffusion chain, alongside the number of details shared with the next person in the chain. This reduced recall early in the chain disadvantaged subsequent people in the chain, in terms of their score on our 'number of details recalled' measure, since they had less information available to recall. To account for this, data were converted into percentages by calculating the proportion of details recalled relative to number of details recalled by the first person in each diffusion chain, and not the number of details in the initial story. Thus, the first participant in each chain would have a proportional score of $100 \%$ and each subsequent participant's score was calculated as a proportion of this (Figure 3).

An ANOVA revealed a main effect of chain type $(F(2.69=11.39, p<0.001)$, see Table 3. Post-hoc contrasts again indicated no significant difference between autistic and non-autistic chains $(\mathrm{MeanA}=63.59, \mathrm{SDA}=24.46$; $\mathrm{MeanN}=58.60, \mathrm{SDN}=24.40$, Tukey HSD $p=0.12$ ). The mixed chain differed significantly from both non-autistic chains (MeanAN $=51.91$, SDAN $=24.54$, Tukey HSD $p<0.05$ ), and autistic chains (Tukey HSD $p<0.001$ ). Regression analysis indicated that there was a steeper decline for the mixed chains $(b=-11.41, S E=5.68$, $p<0.05$ ), while autistic chains' proportion did not differ to that of chains of non-autistic people $(b=5.66, S E=5.68$, $p=0.32$ ), see Table 4 . Position also significantly predicted

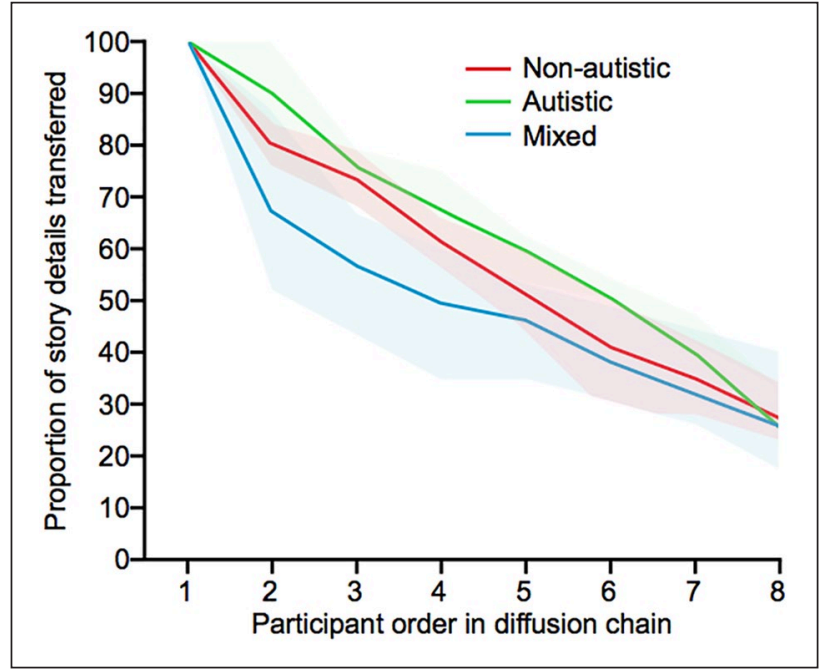

Figure 3. Proportionate mean and range of story details transferred in the diffusion chain, by group and position.

the proportion of information shared $(b=-10.05, S E=0.79$, $p<0.00001$ ), and together chain type and position accounted for $87 \%$ of the variance in proportion of information recalled $(F(5,66)=94.50, p<0.0001, R 2=0.87)$.

Thus, even though the mixed diffusion chains start with a reduced amount of information to transfer, this group still share proportionately less of that information through the diffusion chain. In contrast, autistic and non-autistic chains do not significantly differ from one another in the proportion of information shared.

On completion of the diffusion chain, participants were asked to rate their interactional rapport with each person adjacent to them in the chain, indicating how comfortable they had been during their interactions. Participants rated their rapport both with the person who had recounted the story to them (precede rapport) and the person they had recounted the story to (succeed rapport).

Figure 4 illustrates precede and succeed rapport scores by group. Rapport between chains was compared using Bayesian ANOVA using JASP using a default Cauchy 
Table 4. Regression of the effect of chain type and order in chain on the proportion of data transferred between participants.

\begin{tabular}{|c|c|c|c|c|}
\hline & Estimate & Standard error & $t$ & $p$ \\
\hline Intercept (non-autistic chains) & 103.81 & 4.02 & 25.84 & $<0.0001$ \\
\hline \multicolumn{5}{|l|}{ Main effects } \\
\hline Group: autistic chains & 5.66 & 5.68 & 0.99 & 0.32 \\
\hline Group: mixed chains & $-|1.4|$ & 5.68 & -2.01 & $<0.05$ \\
\hline Order in chain & -10.05 & 0.79 & -12.63 & $<0.0001$ \\
\hline \multicolumn{5}{|l|}{ Interactions } \\
\hline Group: autistic $\times$ order in chain & -0.15 & 1.13 & -0.13 & 0.90 \\
\hline Group: mixed $\times$ order in chain & 1.04 & 1.13 & 0.93 & 0.35 \\
\hline
\end{tabular}

DF: degrees of freedom.

Residual standard error: 8.93 on 66 degrees of freedom. Multiple R-squared $=0.88$; adjusted R-squared $=0.87$. $F$ statistic: 94.50 on 5 and $66 \mathrm{DF}$. $*_{p}<0.0001$.

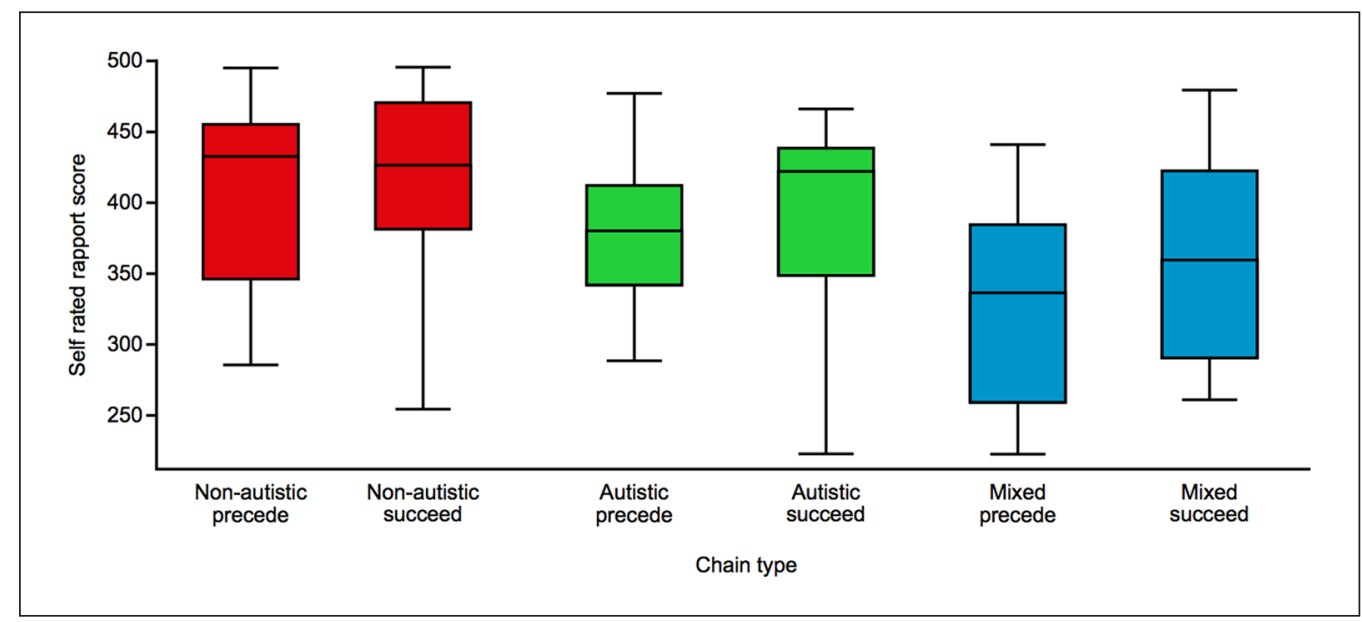

Figure 4. Self-rated interactional rapport by chain type and interaction condition.

prior $(0, r=1 / \operatorname{sqrt}(2))$, and prior odds of 0.59 , and using a Bayes Factor of $<6$ to make inferences as per our preregistration, based on Kass and Raftery (Kass \& Raftery, 1995). Bayesian ANOVA indicated differences between precede rapport in the three groups $(B F 10=71.70$, error $<0.01 \%$ ), with post hoc contrasts showing significant evidence that the non-autistic and mixed chains differed $(\mathrm{BF} 10=62.32$, error $<0.001 \%)$. Evidence of difference was moderate for the comparison of autistic and mixed chains $(\mathrm{BF} 10=5.86$, error $<0.001)$, and there was no evidence of difference in precede rapport between autistic and non-autistic chains $(\mathrm{BF} 10=0.81$, error $<0.01)$. A similar pattern was found for succeed rapport with significant evidence of difference between non-autistic and mixed chains $(B F 10=6.55$, error $<0.001)$, but no evidence of difference between the autistic and mixed group $(\mathrm{BF} 10=0.57$, error $<0.05 \%)$ nor between the autistic and non-autistic groups $(\mathrm{BF} 10=0.67$, error $<0.05 \%)$.

Within this diffusion chain paradigm, autistic people recall information shared by autistic peers as effectively, as non-autistic people recall information shared by non-autistic peers. Yet, information sharing is significantly poorer in chains of mixed neurotypes. These deficits in information transfer between mixed neurotype groups are accompanied by significantly poorer self-rated interactional rapport.

\section{Discussion}

Autism is conceptualised clinically, and in scientific research, by core deficits in social communication, interaction and emotional reciprocity, deficits in non-verbal communicative behaviours used for social interaction and an absence of interest in peers (APA, 2013). In theory, this should translate into poor information transfer with others. These results, however, are the first empirical evidence that suggest the difficulties in autistic communication are apparent only when interacting with non-autistic people, and are alleviated when interacting with autistic people. This is evidenced by our finding that autistic and nonautistic people do not significantly differ in how accurately they recall information from peers of the same neurotype but that selective difficulties occur when autistic and nonautistic people are sharing information. This occurs alongside significantly lower rapport within mixed groups. 
These results challenge traditional assumptions of autistic social impairment. The findings are inconsistent with the social-cognitive deficit narrative of autism. We found a selective breakdown of information transfer and rapport occurred in mixed autistic-non-autistic interactions, indicating that the diagnostic status of an interlocutor plays a critical role in both the quality and enjoyment of and interaction, for both autistic and non-autistic people. The quality of transfer of information within all autistic chains did not differ from information transfer in all nonautistic chains, indicating that autistic peoples' abilities to share information and build rapport do not significantly differ from their non-autistic counterparts.

These findings are consistent with prior research indicating that autistic people experiencing close social bonds and empathy with other autistic people, though may experience specific difficulty interacting with non-autistic people (Crompton et al., 2019; Morrison et al., 2019). This lends additional support to the Double Empathy theory, and suggests that autistic social 'deficits' are better conceptualised as interaction and communicative challenges, operating bi-directionally for autistic and non-autistic people (Fletcher-Watson \& Bird, 2020; Milton, 2012). Our data are reminiscent of established effects of in-group/outgroup status on imitation, suggesting that autistic people are different without being deficient (Bourgeois \& Hess, 2008; Yabar et al., 2006) and have implications for autism diagnosis and post-diagnostic support.

This study raises many questions for future research; for example, would mixed chains show a different pattern if an autistic participant began the chain? We could hypothesise that the first non-autistic participant in the mixed chain is recalling and sharing fewer details due to an anxiety around how to interact with autistic people, or an intentional oversimplification of information for the perceived benefit of the autistic learner. These effects may or may not be present if an autistic person were the first participant in the chain, and future research may focus on this question. However, if it were the case that non-autistic people oversimplify in an attempt to lower communicative pressures on autistic people, this could have a dramatic impact on autistic peoples' ability to get the information they need from non-autistic people in their day-to-day life. Future work should focus on understanding information transfer, rapport and dynamics between mixed pairs and groups, as well as in within autistic groups, to more fully understand the mechanisms underlying autistic social cognition.

This study does have limitations, which could be addressed by future research in this field. First, though fully powered to detect even moderate effects, the sample size was relatively modest, and replications are warranted. Second, participants were aware of the diagnostic status of the person with whom they were interacting, which could have affected their behaviour. However, previous research has shown that when non-autistic people believe they are interacting with an autistic person, they attempt to behave in a helpful way (Heasman \& Gillespie, 2019b), and sharing diagnostic information results in greater acceptance of autistic people (Sasson \& Morrison, 2017). As such, it may be hypothesised that an even larger effect would be found if participants were blind to the diagnostic status of the others in their diffusion chain. Third, the diffusion chains were not equally divided by gender which may have affected results (Wood et al., 2013). However, the most prominent comparisons of autistic males and autistic females to date have focused on access to diagnosis (Rutherford et al., 2016), co-occurring conditions (Mannion et al., 2013) and behavioural profiles (Kirkovski et al., 2013), but not on factors expected to drive this effect, for example, recall ability or verbal IQ. Thus, we cannot hypothesise whether the gender distribution in this sample drives the effect via cognitive mechanisms. It is possible that rapport scores and social dynamics could be different within a more mixed or predominantly male sample, for example, if autistic males were less likely to camouflage (Hull et al., 2020) and are thus more likely to align with non-autistic expectations of what autism is. While this is a tentative hypothesis, if anything this could lead to an even greater disadvantage in the mixed chains. Fourth, the samples had IQ within the typical range, communicated verbally and were likely to have received their diagnosis in adulthood given the relatively high mean age of diagnosis. It is not known whether similar effects would be found in autistic people with intellectual disability.

Finally, the Bayesian comparison for rapport did not yield especially high Bayes Factors when comparing autistic and mixed groups. Further work needs to establish the validity and reliability of this effect through replication and across tasks and populations. Specifically, the connection between poor information transfer and lower ratings of rapport bears further scrutiny - does lack of rapport drive the effects we've seen here? Or is the mechanism more behavioural, for example, due to mismatches in the type and manifestation of social cues used during the interactional task? Uncovering the mechanism of the effects reported here will have probable implications for our understanding of social cognition and interaction itself (Sheppard et al., 2016).

In addition to these conceptual advances, our findings may also carry relevant practical implications for psychological, psychiatric and social practice. Confirmation of the finding that autistic social difficulties operate solely across the autistic-non-autistic divide could have profound implications for the classification of autism as a disorder in the Diagnostic and Statistical Manual (APA, 2013). In the meantime, our data suggest that (non-autistic) practitioners supporting autistic people should be conscious of the challenges to information-transfer described here. In the context of rising concern about suicide in autism (Cassidy \& Rodgers, 2017) and evidence that 'sense of belonging' is a 
key protective factor against suicide (Pelton \& Cassidy, 2017) evidence of improved rapport between autistic people bolsters existing calls for more autistic peer-to-peer support (Iemmi et al., 2017). Subsequent research is required to delineate the differences in autistic and non-autistic interaction styles, which will offer practical utility to psychologists, psychiatrists and health professionals, if they can be taught to adapt their communication style to better accommodate people with autism. While replications are warranted, this radical finding challenges the way autism has been characterised for decades, and there are significant and wide-reaching implications for how autistic people are supported in society.

\section{Acknowledgements}

The authors thank Felicity Sedgewick for her input with task design, and Panagiota Ira Bitsola, Sakura Brandi, Laura Carney, James Clarke and Alice Hodgkinson for their assistance during data collection.

\section{Declaration of conflicting interests}

The author(s) declared no potential conflicts of interest with respect to the research, authorship and/or publication of this article.

\section{Funding}

The author(s) disclosed receipt of the following financial support for the research, authorship, and/or publication of this article: This study was supported by a grant from the Templeton World Charity Foundation (grant number: TWCF0200).

\section{ORCID iDs}

Catherine J Crompton (iD https://orcid.org/0000-0001-5280-1596

Sue Fletcher-Watson (iD https://orcid.org/0000-0003-2688-1734

\section{Supplemental material}

Supplemental material for this article is available online.

\section{References}

American Psychiatric Association. (2013). Diagnostic and Statistical Manual of Mental Disorders (DSM-5®). American Psychiatric Publishing.

Baron-Cohen, S., Jolliffe, T., Mortimore, C., \& Robertson, M. (1997). Another advanced test of theory of mind: Evidence from very high functioning adults with autism or Asperger syndrome. Journal of Child Psychology and Psychiatry, 38, 813-822.

Baron-Cohen, S., O’Riordan, M., Stone, V., \& Jones, R. (1999). Recognition of faux pas by normally developing children and children with Asperger syndrome or high-functioning autism. Journal of Autism and Developmental Disorders, 29, 407-418.

Baron-Cohen, S., Wheelwright, S., Skinner, R., Martin, J., \& Clubley, E. (2001). The autism-spectrum quotient (AQ): Evidence from Asperger syndrome/high-functioning autism, males and females, scientists and mathematicians. Journal of Autism and Developmental Disorders, 31, 5-17.

Bartlett, F. (1932). Remembering: A study in experimental and social psychology. Cambridge University Press.

Bourgeois, P., \& Hess, U. (2008). The impact of social context on mimicry. Biological Psychology, 77, 343-352.

Cassidy, S., \& Rodgers, J. (2017). Understanding and prevention of suicide in autism. The Lancet Psychiatry, 4(6), e11.

Crompton, C. J., \& Fletcher-Watson, S. (2019). Investigating the transfer of information in neurodiverse groups. Open Science Framework. https://osf.io/krbsz/

Crompton, C. J., Hallett, S., Ropar, D., Flynn, E., \& FletcherWatson, S. (2020). 'I never realised everybody felt as happy as I do when I am around autistic people': A thematic analysis of autistic adults' relationships with autistic and neurotypical friends and family. Autism. https://doi. org/10.1177/1362361320908976

DeBrabander, K. M., Morrison, K. E., Jones, D. R., Faso, D. J., Chmielewski, M., \& Sasson, N. J. (2019). Do first impressions of autistic adults differ between autistic and nonautistic observers? Autism in Adulthood, 1(4), 250-257.

Edey, R., Cook, J., Brewer, R., \& Johnson, M. (2016). Interaction takes two: Typical adults exhibit mind-blindness towards those with autism spectrum disorder. Journal of Abnormal Psychology, 125, 879-885.

Flesch, R. (1948). A new readability yardstick. Journal of Applied Psychology, 32, 221-233.

Fletcher-Watson, S., \& Bird, G. (2020). Autism and empathy: What are the real links? Autism, 24(1), 3-6.

Gernsbacher, M. A., Stevenson, J. L., \& Dern, S. (2017). Specificity, contexts, and reference groups matter when assessing autistic traits. PLOS ONE, 12(2), Article e0171931.

Heasman, B., \& Gillespie, A. (2018). Perspective-taking is twosided: Misunderstandings between people with Asperger's syndrome and their family members. Autism, 22(6), 740-750.

Heasman, B., \& Gillespie, A. (2019a). Neurodivergent intersubjectivity: Distinctive features of how autistic people create shared understanding. Autism, 23, 910-921.

Heasman, B., \& Gillespie, A. (2019b). Participants over-estimate how helpful they are in a two-player game scenario toward an artificial confederate that discloses a diagnosis of autism. Frontiers in Psychology, 10, Article 1349.

Hull, L., Lai, M. C., Baron-Cohen, S., Allison, C., Smith, P., Petrides, K. V., \& Mandy, W. (2020). Gender differences in self-reported camouflaging in autistic and non-autistic adults. Autism, 24, 352-363.

Iemmi, V., Knapp, M., \& Ragan, I. (2017). The autism dividend: Reaping the rewards of better investment. National Autism Project. http://nationalautismproject.org.uk/wp-content/ uploads/2017/01/autism-dividend-report.pdf

JASP Team. (2019). JASP (Version 0.10.1). https://jasp-stats. org/

Kass, R. E., \& Raftery, A. E. (1995). Bayes factors. Journal of the American Statistical Association, 90, 773-795.

Kenny, L., Hattersley, C., Molins, B., Buckley, C., Povey, C., \& Pellicano, E. (2016). Which terms should be used to describe autism? Perspectives from the UK autism community. Autism, 20(4), 442-462.

Kincaid, J. P., Fishburne, R. P., Jr., Rogers, R. L., \& Chissom, B. S. (1975). Derivation of new readability formulas 
(automated readability index, fog count and Flesch reading ease formula) for navy enlisted personnel (Institute for Stimulation and Training Research Reports, 56). https:// stars.library.ucf.edu/istlibrary/56

Kirkovski, M., Enticott, P. G., \& Fitzgerald, P. B. (2013). A review of the role of female gender in autism spectrum disorders. Journal of Autism and Developmental Disorders, 43(11), 2584-2603.

Mannion, A., Leader, G., \& Healy, O. (2013). An investigation of comorbid psychological disorders, sleep problems, gastrointestinal symptoms and epilepsy in children and adolescents with autism spectrum disorder. Research in Autism Spectrum Disorders, 7(1), 35-42.

Mesoudi, A., \& Whiten, A. (2008). The multiple roles of cultural transmission experiments in understanding human cultural evolution. Philosophical Transactions of the Royal Society B: Biological Sciences, 363, 3489-3501.

Milton, D. E. M. (2012). On the ontological status of autism: The 'double empathy problem'. Disability \& Society, 27, 883-887.

Milton, D. E. M., Heasman, B., \& Sheppard, E. (2018). Double empathy. In F. Volkmar (Ed.), Encyclopedia of autism spectrum disorders (pp. 1-8). Springer.

Morrison, K. E., DeBrabander, K. M., Jones, D. R., Faso, D. J., Ackerman, R. A., \& Sasson, N. J. (2019). Outcomes of real-world social interaction for autistic adults paired with autistic compared to typically developing partners. Autism. https://doi.org/10.1177/1362361319892701

Pelton, M. K., \& Cassidy, S. A. (2017). Are autistic traits associated with suicidality? A test of the interpersonal-psychological theory of suicide in a non-clinical young adult sample. Autism Research, 10(11), 1891-1904.

Persicke, A., Tarbox, J., Ranick, J., \& Clair, M. (2013). Teaching children with autism to detect and respond to sarcasm. Research in Autism Spectrum Disorders, 7, 193-198.
Ritvo, R., Ritvo, E., Guthrie, D., Ritvo, M., Hufnagel, D. H., McMahon, W., ... Eloff, J. (2011). The Ritvo Autism Asperger Diagnostic Scale-Revised (RAADS-R): A scale to assist the diagnosis of autism spectrum disorder in adults: An international validation study. Journal of Autism and Developmental Disorders, 41, 1076-1089.

Rutherford, M., \& Baron-Cohen, S. (2002). Reading the mind in the voice: A study with normal adults and adults with Asperger syndrome and high functioning autism. Journal of Autism and Developmental Disorders, 32, 189-194.

Rutherford, M., McKenzie, K., Johnson, T., Catchpole, C., O'Hare, A., McClure, I., . . . Murray, A. (2016). Gender ratio in a clinical population sample, age of diagnosis and duration of assessment in children and adults with autism spectrum disorder. Autism, 20(5), 628-634.

Sasson, N. J., Faso, D. J., Nugent, J., Lovell, S., Kennedy, D. P., \& Grossman, R. B. (2017). Neurotypical peers are less willing to interact with those with autism based on thin slice judgments. Scientific Reports, 7, 40700.

Sasson, N. J., \& Morrison, K. E. (2017). First impressions of adults with autism improve with diagnostic disclosure and increased autism knowledge of peers. Autism, 23, 50-59.

Sheppard, E., Pillai, D., Wong, T.-L., Ropar, D., \& Mitchell, P. (2016). How easy is it to read the minds of people with autism spectrum disorder? Journal of Autism and Developmental Disorders, 46, 1247-1254.

Wechsler, D. (2011). WASI-II: Wechsler Abbreviated Scale of Intelligence (2nd ed.). Psychological Corporation.

Wood, L. A., Kendal, R. L., \& Flynn, E. G. (2013). Whom do children copy? Model-based biases in social learning. Developmental Review, 33, 341-356.

Yabar, Y., Johnston, L., Miles, L., \& Peace, V. (2006). Implicit behavioral mimicry: Investigating the impact of group membership. Journal of Nonverbal Behavior, 30, 97-113. 